\title{
Clinical Chalenges in Differential Diagnosis of Oral Lesions
}

\author{
Elitsa Deliverska ${ }^{1}$, Payam Forghani ${ }^{2}$, \\ Aaron Kooner ${ }^{2}$
}

1. Department of Dental, Oral and Maxillofacial surgery, Faculty of Dental Medicine, Medical University of Sofia;

2. Student in Faculty of Dental Medicine, Medical University of Sofia;

\begin{abstract}
Differential diagnosis of oral mucosal lesions is primarily evaluated using a diagnosis algorithm or protocol, together with the clinical presentation of the lesion, anamnesis of the patient and the clinical knowledge of the clinician. This review has been designed to investigate the most prevalent lesions and phenomena of the oral mucosa, and to guide the clinician to correct and efficient approach in the diagnosis of such lesions. The success of a rapid and correct diagnosis of oral mucosal lesions is of great advantage for the patient, ensuring timely management and correct treatment procedures. Most importantly, the timely detection and exclusion of malignant lesions upon a routine dental oral examination provide the clinician and patient with maximum success in treatment opportunities.
\end{abstract}

Keywords: oral lesions, oral mucosa, diagnosis, clinical features

\section{Background}

The abundance of various oral mucosal lesions, and their differentiation in clinical appearance, localisation, symptoms and treatment, requires precise diagnosis by the clinician in order to maximise therapeutic success. The diagnosis of oral mucosal lesions and their distinction from other conditions may be best 
accomplished by using a diagnostic algorithm. $(1,2)$ Relevant patient history including anamnesis, clinical behaviour of lesions, and response to any previous treatments, medical history, a complete list of current and recent medications, food sensitivities, allergies, social and family anamnesis. Detailed information of the patients age at onset, number of lesions, severity and period of lesions, recurrence patterns, and factors enhancing or preventing lesion development should be acquired. (3)

Differential diagnosis is is the cognitive process of applying logic al knowledge, in a series of step-by-step decisions, to create a list of possible diagnoses on the basis of exclusion. The clinician can order tests and other diagnostic procedures to narrow the diagnosis.

Clinical presentation should carefully be assessed in terms of location, number, appearance, (including the presence or absence of vesicles, colour, and texture of the lesion borders), duration, degree of discomfort and disability and presence of any extraoral signs and symptoms. Laboratory studies are helpful in establishing if the lesion is associated with a systemic disease when a relationship is suspected. Furthermore, possible iatrogenic factors should also be explored in history taking, sharp margins of dental restorations and poorly fitting prosthetic appliances may also irritate the mucosa and hence be a plausible etiological factor for any given oral mucosal lesion. (4)

The first decision made by the clinician is whether the lesion is a surface lesion or soft tissue enlargement. Surface lesions are lesions that involve the epithelium and superficial connective tissue of the mucosa and skin. Normally they do not exceed 2-3 mm in thickness. Oral lesions can be classified into four groups comprising of ulcerations, pigmentations, exophytic lesions, and red-white lesions. Surface lesions are split into three categories based on their clinical appearance: white, pigmented, and vesicular ulcerated erythematous.

White surface lesions constitute only $5 \%$ of oral pathoses, a few of these lesions such as Leukoplakia, proliferative verrucous leukoplakia and Lichen planus, have malignant potential as high as $0.5-100 \%$. (5) Diagnosis of oral white lesions of oral mucosa might be quite challenging for the clinician. White surface lesions support the appropriate clinical diagnostic approach to exclude the possibility of malignancy. White surface lesions can be categorized according to if they can be scraped off or not and are divided into three groups based on their clinical features:

1. White lesions due to epithelial thickening

2. White lesions due to accumulation of necrotic debris on the mucosal surface

3. White lesions due to subepithelial changes in the connective tissue.

White lesions due to epithelial thickening include lesions which are asymptomatic, rough to palpate, cannot be scraped off and are fixed to the surface must be further examined to see if they have a specific pattern or not and include: Lichen planus, Hairy tongue, leukoplakia, Nicotine stomatitis, Leukoedema, White sponge nevus, Hyperkeratosis, geographic tongue and Squamous cell carcinoma.(6)

White surface lesions due to accumulation of necrotic debris on the mucosal surface are associated with necrosis of the overlying epithelium and can be scraped off. In these surface debris white lesions necrotic epithelium can be removed with a gauze leaving an erythematous or ulcerated base. They are lesions usually painful and include: Oral burns, fibrin clot, Pseudomembranous candidiasis, Materia alba and Morsicatio. 
Pigmented surface lesions of oral mucosa appear blue, brown, or black. They could be classified as generalized lesions( diffuse and multifocal), and localized lesions, which usually are unilateral and involve only one or several locations.

Localized pigmented lesions of oral mucosa are categorized into four subgroups(7):

- Intravascular blood lesions which include: Varix, Kaposi's Scarcoma and Hemangioma, these lesions usually go pale on pressure and can be compressed.

- Extravascular blood lesions which include: Hematoma, Petechiae and Ecchymosis.

- Melanocytic lesions which include: Melanocytic nevus, Melanoma, Oral melanotic macule, and Ephelis

- Tattoo lesions.

Benign pigmented surface lesions show regular borders, are small, uniform in colour and symmetrical, they can be either flat or slightly elevated. In contrast malignant pigmented surface lesions show irregular borders, surface ulceration and colour variation. Clinical tests such as radiography, diascopy and laboratory investigations such as blood tests can be used to confirm a clinical theory and reach a definitive diagnosis. Oral pigmentation is common, and its differential diagnosis is vast. (8) The pigmentation can be the result of various exogenous factors like embedded foreign material such as tattoo pigment, pencil lead or amalgam from dental restorations. Endogenous pigmentation can be associated with numerous syndromes and physiological conditions, including Addison's disease, Peutz-Jeghers syndrome and Laugier-Hunziker syndrome. (9) Because of the different appearance of oral pigmented lesions, diagnosis is not always made reliably by a single examination. Clinicians should therefore have a low threshold to biopsy(in melanoma cases should not be performed incisional biopsy, only cytology test could be performed) on these important lesions.

Generalized Pigmented Surface Lesions of Oral Mucosa are bilateral, multiple and diffuse (hereditary, pregnancy, due to medication, smoker's melanosis, heavy metals melanosis, neurofibromatosis, Addison disease.

Vesicular ulcerated erythematous are categorized into solitary acute, multiple acute, solitary chronic, multiple chronic, and recurrent lesions. The first decision to be made is whether the ulcerative lesion is of an acute, chronic, or recurrent nature. After that, the lesions should be placed in one of the following five subgroups (10-13):

1. Solitary acute lesions are mostly identified as Traumatic ulcers and Necrotising sialometaplasia.

2. Multiple acute lesions occur in clusters and may be primary Herpetic gingivostomatitis, Varicella zoster infection, Herpangina, Hand-foot-and mouth disease, Erythema multiforme, Necrotising ulcerative gingivitis or Oral hypersensitivity reactions. Solitary chronic lesions include TB ulcers, Syphilitic chancres, Ulcerative SCC, Chronic traumatic ulcers and possibly Necrotising sialometaplasia.

3. Multiple chronic lesions, which occur in groups, include Lichen planus, Pemphigus and Pemphigoid ulcers.

4. Recurrent lesions, including Recurrent aphthous stomatitis, Recurrent herpes stomatitis, Cyclic neutropenia and Behcet's disease. 
A Nikolsky sign may be present in epidermolysis bullosa, pemphigus, mucous membrane pemphigoid, lichen planus and lupus erythematosus but is not positive in all patients with these diseases. Erythema multiforme and medication- induced mucositis, contact stomatitis should be considered as a differential diagnosis.

White lesions due to subepithelial changes in the connective tissue have normal overlying epithelium and changes in the connective tissue can mask blood vessels and cause the area to appear with different colour: white, yellow or tan. These lesions usually have a smooth translucent surface are not painful and can not be scraped off (gingival cyst of the newborn, Fordyce granules, subepithelial fibrosis, scar).

Soft tissue enlargements are growths or masses that are divided into two categories: reactive and tumours (neoplasms). Reactive soft tissue enlargements are regressive, resolve, often symptomatic, fluctuate in size, are often tender or painful and usually have a more rapid growth rate measured in hours to weeks than tumours. Some of the reactive enlargements begin as a diffuse lesion and become more localized with time. Sometimes reactive lesions are associated with tender lymph nodes and systemic manifestations, such as fever and malaise. If it is decided that a soft tissue enlargement is reactive lesion, the next step is to determine what the lesion is reacting to, such as chronic irritation, bacterial, fungal or viral infections or chemical and physical injury. (14)

Soft tissue tumours/neoplasms/ are persistent and progressive, often asymptomatic, have a growth rate of weeks to years and do not resolve without treatment. The Irritation fibroma is classified as a tumour because it is persistent and progressively increases in size, although generally clinicians agree that the correct pathogenesis is that of a reactive process secondary to chronic irritation. $(15,16)$ It is very important for tumours/ neoplasms to be diagnosed as early as possible. Although removal of the lesion and microscopic examination of the tissue is the only way to arrive at a definitive diagnosis, there are some basic principles which may aid the clinician in the correct diagnosis of the lesion. If a soft tissue enlargement appears to be a tumour, the clinician must determine if the enlargement is benign or malignant.

Benign tumours are usually well defined, circumscribed and have a slower growth rate of months and years. Some benign tumours are fixed to surrounding structures, but other benign tumours are surrounded by a fibrous connective tissue capsule, which may allow the lesion to be moved within the tissue independent of surrounding structures. $(17,18)$

Benign tumors are subdivided into four categories: Epithelial tumors which include Verruca vulgaris, Papilloma and Condyloma acuminatum, Keratoakantoma, Cornu cutaneum; Mesenchymal tumors which include Irritation fibroma, Epulis fissuratum, Rabdomyoma, Leiomyoma, Hemangioma, Lymphangioma, Lipoma etc. Salivary gland tumors which include, Pleomorphic adenoma, Oncocytoma etc.

Differential diagnosis must be done with lymphadenopathy and cysts of soft tissue which include: Gingival cyst, Thyroglossal tract cyst, branchial, Nasolabial cyst etc. Although these soft tissue lesions are not tumours, their clinical features resemble those of benign tumours. $(19,20)$

Malignant neoplasms are more prone to being painful and cause ulceration of the overlying epithelium(especially these of epithelium origin). Since malignant neoplasms invade or infiltrate surrounding muscle, blood vessels, nerve and connective tissue, they are fixed to surrounding structures during palpation. $(21,22)$ Malignant neoplasms include Lymphomas, melanoma, from epithelium and mesenchimal 
origin include: squamous cell carcinoma, salivary gland adenocarcinoma, mucoepidermoid, adenocystic $\mathrm{Ca}$, fibrosarcoma, liposarcoma, leiomyosarcoma etc. Pathohystological examination should be performed as soon as the lesion was detected.

\section{Conclusion}

Together with the exact anamnesis evaluation and careful inspection of the clinical presentation of an oral mucosal lesion, the use of a diagnosis decision algorithm can help the clinician to be directed to a more precise diagnosis. Although there exist some limitations to the scope and capabilities of such a decision algorithm, it is important to eliminate completely and thoroughly other irrelevant and implausible differential diagnoses. Both clinicians and patients can take advantage of decision protocols, as an improved treatment success and the out-patient outcome relies on early, precise diagnosis.

\section{References}

1. Baccaglini L, Theriaque D W., Shuster J J., Serrano G, Lalla, R V. Validation of anamnestic diagnostic criteria for recurrent aphthous stomatitis, J Oral Pathol Med (2013) 42: 290-294

2. Finkelstein, M W, Lanzel, E. A Guide to Clinical Differential Diagnosis of Oral Mucosal Lesions, on line course, www.dentalcare.com/en-us/professional-education/ce-courses/ce110

3. Kauzman, A, Pavone, M, Blanas, N, Bradley, G. Pigmented Lesions of the Oral Cavity: Review, Differential Diagnosis, and Case Presentations, J Can Dent Assoc 2004; 70(10):682-3

4. Kovac-Kavcic, M, Skaleric, U. The prevalence of oral mucosal lesions in a population in Ljubljana, Slovenia, J Oral Pathol Med 2000: 29: 331-5

5. Silverman S, Mucosal lesions in older adults, JADA 2007;138(9 supplement):41S-46S.

6. Nevalainen, M J, Narhi T O, Ainamo, A. Oral mucosal lesions and oral hygiene habits in the home-living elderly, Journal of Oral Rehabilitation 19971997 May;24(5):332-7.

7. Müller, S Melanin-associated pigmented lesions of the oral mucosa: presentation, differential diagnosis, and treatment, Dermatologic Therapy, Vol. 23, 2010, 220-229

8. Powell, J P, Cummings, C W. MD, Melanoma and the differential diagnosis of oral pigmentated lesions, The laryngoscope 1978 Aug;88(8 Pt 1):1252-65

9. Bolewska, J, Hansen, H J DDS, Holmstrup, Palle, Pindborg, J J, Stangerup, M. Oral mucosal lesions related to silver amalgam restorations, ORAL SURC ORAL MED ORAL PATHOL, 1990 Jul;70(1):55-8

10. Espinoza,I, Rojas, R. Aranda, W. Gamonal, J. Prevelence of oral mucosal lesiona in elderly people in Santiago, Chile, J Oral Pathol Med 2003 Nov;32(10):571-5.

11. Messadi D V, Younai, F. Aphthous ulcers, Dermatologic Therapy, 2010, Vol. 23, 281-290

12. Herman, W W. Konzelman, J L, Thompson, A L , Bonta, C Y , Update on Recurrent Aphthous Stomatitis. Pract Hyg 2002;11(6):35-38.

13. Femiano, F, Lanza, A, Buonaiuto, C, Gombos, F, Cirillo, N. Oral aphthous-like lesions, PFAPA syndrome: a review, J Oral Pathol Med (2008) 37: 319-323

14. Lambertini M, Patrizi, A, Ravaioli, GM, Dika, ME. Oral pigmentation in physiologic conditions, post-inflammatory affections and systemic diseases G Ital. Dermatol Venerol. 2018 Oct;153(5):666-671. doi: 10.23736/S0392-0488.17.05619-X. 19.

15. Reichart PA, Philipsen HP: Betel chewer's mucosa - a review. J Oral Pathol Med 1998; 27: 239-42.

16. Fernandes R, Tuckey T, Lam P, Allidina S, Sharifi S, Nia D. The Best Treatment For Aphthous Ulcers J Am Dent Ass. 2008;134:200-7. 
17. Yeatts D, Burns, J C. Common Oral Mucosal Lesions in Adults,American Family Physician, dec 1991, vol 44, no 6, 2043-2050

18. Mravak-Stipetić, M. Differential diagnostics of painful conditions of oral mucosa, Rad 507. Medical Sciences, 34(2010):55-73

19. Ozkaya, E. Oral mucosal fixed drug eruption: Characteristics and differential diagnosis L Am Acad Dermatol. 2013 Aug;69(2):e51-8. doi: 10.1016/j.jaad.2012.08.019.

20. Giovannelli L, Campisi, G, Lama,A, Giambalvo,O, Osborn, J, Margiotta, V, Ammatuna, P.Human Papillomavirus DNA in Oral Mucosal Lesions, The Journal of Infectious Diseases, Volume 185, Issue 6, 15 March 2002, Pages 833-836, https://doi.org/10.1086/339193

21. Holmstrup P, Oral mucosa and skin reactions related to amalgam, Adv Dent Res 1992 Sep;6:120-4.

22. Budtz-Jorgensen, E. Oral mucosal lesions associated with the wearing of removable dentures, Journal of Oral Pathology 1981 Apr;10(2):65-80.

\section{Corresponding author:}

Associate prof. Elitsa Deliverska, PhD,

Faculty of Dental Medicine,

Department of Dental, Oral and Maxillofacial Surgery,

Medical University of Sofia

1 Sveti Georgi Sofiiski str.,

1431 Sofia, Bulgaria 